\title{
Multi-platform experiment to cross a boundary between laboratory and real situational studies: experimental discussion of cross-situational consistency of driving behaviors
}

\author{
Terai, H. ${ }^{\mathrm{a}, \mathrm{e}^{*}}$, Miwa, K. ${ }^{\mathrm{a}}$, Okuda, H. ${ }^{\mathrm{b}, \mathrm{e}}$, Tazaki, Y. ${ }^{\mathrm{b}}$, Suzuki, T ${ }^{\mathrm{b}}$, Kojima, K. ${ }^{\mathrm{c}}$, Morita, J. ${ }^{\mathrm{d}}$, Maehigashi, \\ A. $^{\mathrm{a}}$, and Takeda, $\mathrm{K}$. ${ }^{\mathrm{a}}$ \\ ${ }^{a}$ Graduate School of Information Science, Nagoya University, Furo-cho, Chikusa-ku, Nagoya, Aichi, Japan \\ ${ }^{\mathrm{b}}$ Graduate School of Engineering, Nagoya University, Furo-cho, Chikusa-ku, Nagoya, Aichi, Japan \\ ${ }^{\mathrm{c}}$ Faculty of Human Sciences, Waseda University, 2-579-15, Mikajima, Tokorozawa, Saitama, Japan \\ ${ }^{\mathrm{d} J a p a n}$ Advanced Institute of Science and Technology, 1-1, Asahidai, Nomi, Ishikawa, Japan \\ 'Japan Science and Technology Agency, Core Research for Evolutional Science and Technology, 4-1-8, Hon- \\ machi, Kawaguchi, Saitama, Japan
}

\begin{abstract}
We constructed an innovative experimental platform to study cross-situational consistency in driving behavior, conducted behavioral experiments, and reported the data obtained in the experiment. To discuss cross-situational consistency, we separated situations in which people use some systems to conduct tasks into three independent conceptual factors: environment, context, and system. We report the experimental results with the following systems: a laboratory system with a gaming controller and steering/pedal controllers and a real system, COMS an instrumented vehicle. The results are summarized as follows. 1) The individual behaviors in each system were stable, and consistency was retained. 2) The consistency of the behaviors was also confirmed when the participants drove using different interfaces in identical systems. 3) However, only slight correlation was observed across different systems in a specific situation where a strong high-order cognitive constraint (i.e., rapid driving) and a weak low-order cognitive constraint (driving with easy handling toward a straight-line course) were given.
\end{abstract}

Keywords: behavior analysis; cross-situational consistency; driving behavior; laboratory studies; multi-environment; real situational studies

\section{Introduction}

The consistency of human behavior is an important assumption for conducting cognitive and behavioral studies. Many arguments such as personsituation and consistency debates have discussed the effects on consistency of a variety of situation factors and human individualities (e.g., [9]).

In laboratory studies with humans, such consistency has been discussed from the viewpoint of ecologi- cal validity and external validity $[3,8]$. For example, Anderson, Lindsay, and Bushman (1999) showed correlations between the results of laboratory studies and field studies and discussed the psychological reality of laboratory findings by conducting metaanalysis especially for individuality and social behaviors [1].

On the other hand, in applied studies related to human system interaction, system usage behaviors and their usability are evaluated using virtual systems

*E-mail: terai@is.nagoya-u.ac.jp 
on behalf of real systems because ethical as well as technical constraints restrict the margin of real-world studies. For example, driving simulator replicates highly driving environment with ability of repetition and controllability [6]. There is a diverse range of studies about driving errors, reaction time, and cognitive performance to reveal the absolute and relative validity of driving simulators (e.g., [4-5]).

As described above, the effect of situation and personality on behavioral consistency has been discussed in a wide variety of research areas, including psychological and applied studies. In recent years, the importance of discussing such consistency based on behavioral data continues to increase. However, there are few unified views as a cross cutting perspective across a wide range of ecological validity based on behavioral experiments [2, 7].

Additionally, for achieving a specific goal, humans usually use electrical and mechanical systems. Empirical data on the consistency retained in human behavior when using different systems may contribute crucial insights in the studies of human factors.

\section{Purpose}

In this study, we constructed an innovative experimental platform to discuss cross-situational consistency in driving behavior, conducted behavioral experiments, and report the data obtained in the experiment.

To discuss cross-situational consistency, we separated situation in which people use some systems to conduct some tasks into three independent conceptual factors: environment, context, and system. The environmental factor determines the physical characteristics in an individual situation and relates to loworder cognitive constraints determining the possible sets of actions. The contextual factor determines the cognitive requirements given from top-down orders or desires in an individual situation and relates to constraints that affect high-order cognition. The system factor corresponds to various external physical systems with which humans perform a given task.

This paper indicates the configurations of an experimental platform to discuss cross-situational consistency and reports our experimental results to investigate cross-situational consistency in driving behavior.

\section{Experimental platform}

Table 1 summarizes the configurations of the experimental platform, which consists of three above independent conceptual factors, to discuss the crosssituational consistency of driving behavior.

\subsection{Systems}

In our experimental platform, we used three types of systems: real, virtual, and laboratory. Participants engaged in identical vehicle-handling tasks with one of the three systems. The real system has the highest ecological validity to perform the vehicle-handling task. The laboratory system has the lowest ecological validity. The virtual system is located between them.

\subsubsection{Real system}

We used an instrumented vehicle called COMS from Toyota Auto Body ${ }^{1}$ as the real system. COMS was equipped with various sensors to record participant behavior, the car dynamics, and the environmental data. The manipulations of the steering wheel and brake/acceleration pedals were recorded as participant behavioral data. The car dynamics data were gathered from speed, acceleration, and angular velocity triaxial sensors. These data were collected at 2000 $\mathrm{Hz}$. The video cameras (front, downward, and face views) were mounted on the COMS. The front view camera captured the road conditions. The downward view camera directed to the road surface and recorded road tags to identify where and when COMS passed specific course points. The face view camera captured the participant faces and steering control. Time codes were synchronized with the logged sensor and video data.

\subsubsection{Virtual system}

A vehicle motion simulator called CarSim from Virtual Mechanics Corporation ${ }^{2}$ was used as the virtual system. The rearview and side mirrors were embedded in the driver's cockpit with the same interior as a real car. The front field of view was $180^{\circ}$ on three screens. As the rearward view, a wide monitor was placed behind the cockpit. Therefore, the virtual system shares many characteristics with the real system. The manipulations of the steering wheel and brake/acceleration pedals were recorded as participant behavioral data. These data were collected at $100 \mathrm{~Hz}$. The participants were also required to control the simulated vehicle, as in the real system.

\footnotetext{
${ }^{1}$ http://www.toyota-body.co.jp/english/products/ev.html

2 http://carsim.com/
} 


\subsubsection{Laboratory system}

In the laboratory system, stimuli were presented to the participants on a 21 -inch computer screen in a similar way as in a usual laboratory setting. There were many differences between the laboratory system and the real and virtual systems. For example, the appearance of the road configuration was shown as a top-down view, and the vehicle controlled by the participants was depicted as a black dot. The car dynamics consisted of simple reactions for the participant inputs. The participants controlled the black dot using two different types of driving interfaces: steering-wheel and pedals (brake and accelerator) or a gaming pad controller. When the participants input right or left as steering control, the dot moved to the corresponding direction by pixels based on input time.
The accelerator and brake operations also accumulated (decreased) the dot velocity. The participant operation data were collected at $25 \mathrm{~Hz}$.

\subsection{Environments}

The participants controlled their vehicles in an experimental driving course environment. The driving course consisted of three physical configurations: sharp and gentle curves and straight lines.

We use such different environmental conditions as course characteristics to learn how environmental

factors affect behavioral consistency.

\subsection{Contexts}

In the experimental platform, we gave experimen-

Table 1

Overview of Exnerimental Platform

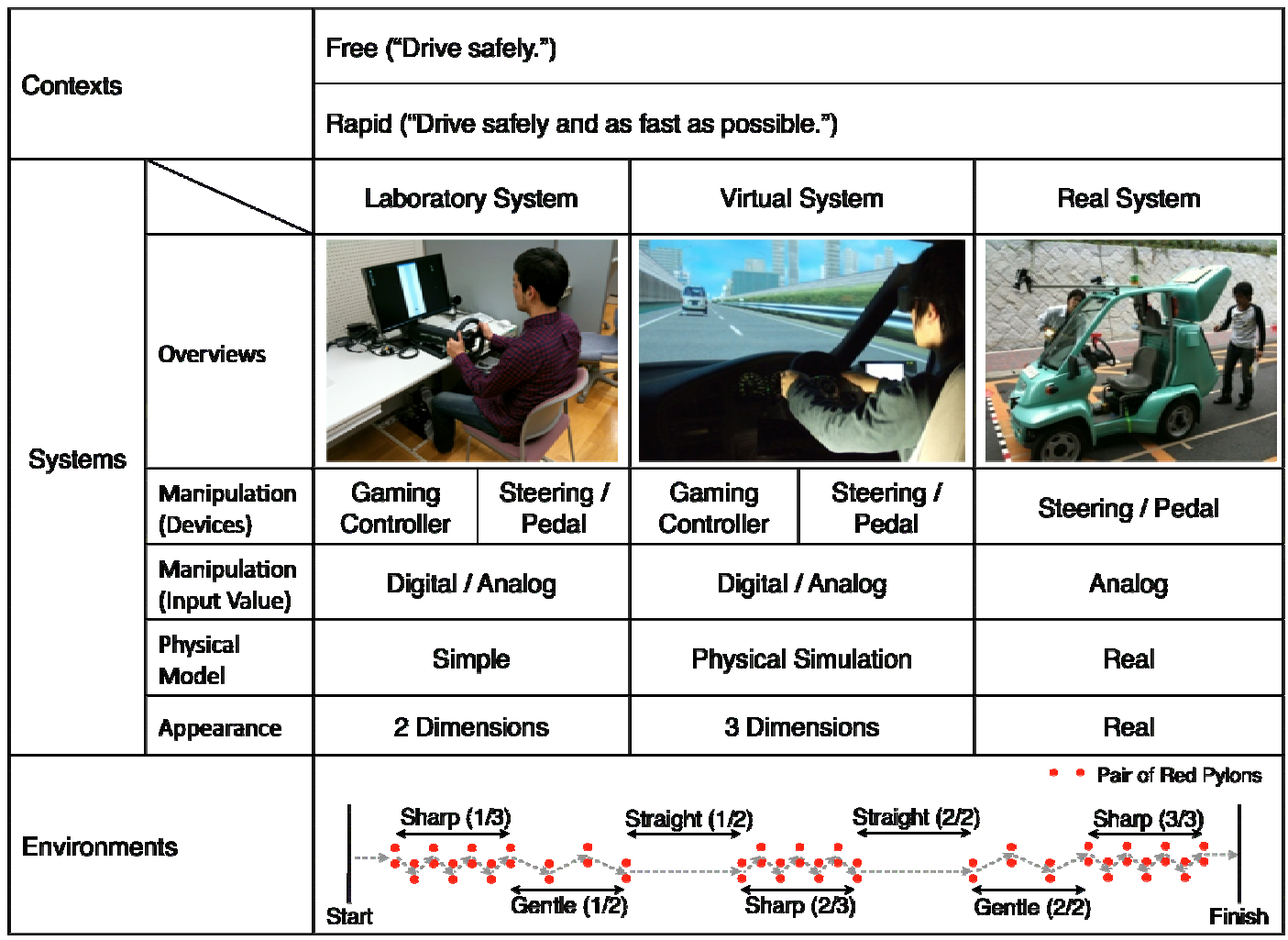

Note. The experimental equipment and settings are summarized from the viewpoint of three dimensions: context, system, and environment. Context corresponds to the instructional requirements related to a high-order cognitive constraint. Environment functions as low-order cognitive constraints and corresponds to the driving course configuration: sharp and gentle curves and straight lines. The course pattern was constructed by adjusting the intervals of pairs of red pylons through which the cars passed (see "environment" in the Table 1). The system consists of three different types of conceptual cars: a gaming car (laboratory system), a simulated car (virtual system), and a real car (real system). 
tal requirements as contextual factors to the participants. There were two different contexts in the driving situation: free driving and rapid driving.

We use such contextual factor to identify how the cognitive requirements given from the top-down orders in an individual situation affect behavioral consistency.

\section{Methods}

In this paper, we report the experimental results where the following were used: a laboratory system with a gaming controller and steering/pedal controllers and a real system, COMS.

\subsection{Participants}

Twenty-one adults (11 males and 10 females) whose ages ranged from 31 to 55 participated. To capture stable vehicle control, they were required to have over ten years of driving experience and to currently drive a car more than ten days a month.

\subsection{Task}

The experimental task assigned to the participants was to drive COMS toward the goal through a course consisting of sharp curves, gentle curves, and straight lines (see environments in Table 1). In our study, the configuration of the driving course corresponds to the environmental factor.

The participants were instructed to drive either as safely or as rapidly as possible. This instruction corresponds to the contextual factor.

In the laboratory system, the participants also engaged in the same driving task as those in the real system.

\subsection{Procedure}

The participation order in each experiment where one of the three systems was utilized was counterbalanced among the participants. In each system, the participants engaged in two sessions, each of which consists of six free driving trials and six rapid driving trials. First, the participants drove on the experiment course twice as preliminary practice. After the practice session, they engaged in six free driving trials where only safe driving was required. They also engaged in six rapid driving trials where they were instructed to drive as fast as possible improving their lap time across the trials while continuing to drive safely. This instruction gave the context to the participants.

\section{Results and discussion}

In this section, we summarize our experimental results from two viewpoints to discuss cross-situational consistency. The first is the behavioral stability of the participants when operating each system (within the system), and the second is the behavioral consistency across the systems.

\subsection{Behavioral stability within system}

Table 2 shows the results of Piason's correlation analysis of individual behaviors in the former and latter three trials in all six trials on each course pattern (see environments in Table 1). In this analysis, we used the average amount of each operation (brake, accelerator, and steering) per sampling point.

The result indicates high correlations in almost all cells in Table 2, meaning that individual behaviors were stable in driving when using each system.

\subsection{Behavioral consistency across systems}

Next, we conducted another correlation analysis between the systems to discuss the cross-situational consistency (Table 3). Significant correlation in the acceleration and steering operations was observed between the two different controllers in the laboratory system. However, we only found slight correlation between the real and laboratory systems. Significant correlation only arose in a very limited situation (highlighted in Table 3): the rapid requirement in the context and the gentle course pattern from the end of the sharp curve to the start of the straight line in the environment. 
Table 2

Behavioral stability within systems: correlations between individual behavior at former half trials and latter

Context Free Driving
Brake operation
\begin{tabular}{|l|c|c|c|}
\hline & Real & Laboratory (Steering \& Pedals) & Laboratory (Game Pad) \\
\hline Sharp $(1 / 3)$ & $r=0.97 *$ & $r=0.74 *$ & $r=0.80 *$ \\
\hline Gentle $(1 / 2)$ & $r=0.97 *$ & $r=0.99 *$ & n.s. \\
\hline Straight $(1 / 2)$ & $r=0.97 *$ & n.s. & $r=0.66 *$ \\
\hline Sharp $(2 / 3)$ & $r=0.97 *$ & $r=0.94 *$ & $r=0.87 *$ \\
\hline Straight $(2 / 2)$ & $r=0.97 *$ & n.s. & n.s. \\
\hline Gentle $(2 / 2)$ & $r=0.97 *$ & $r=0.99 *$ & $r=0.70 *$ \\
\hline Sharp $(3 / 3)$ & $r=0.97 *$ & n.s. & $r=0.78 *$ \\
\hline
\end{tabular}

Context Rapid Driving

Brake operation
\begin{tabular}{|l|c|c|c|}
\hline & Real & Laboratory (Steering \& Pedals) & Laboratory (Game Pad) \\
\hline Sharp $(1 / 3)$ & $r=0.93 *$ & $r=0.89 *$ & $r=0.90 *$ \\
\hline Gentle $(1 / 2)$ & $r=0.96 *$ & n.s. & n.s. \\
\hline Straight $(1 / 2)$ & $r=0.92 *$ & $r=0.53 *$ & $r=0.88 *$ \\
\hline Sharp $(2 / 3)$ & $r=0.94 *$ & $r=0.81 *$ & $r=0.91 *$ \\
\hline Straight $(2 / 2)$ & $r=0.94 *$ & n.s. & n.s. \\
\hline Gentle $(2 / 2)$ & $r=0.97 *$ & n.s. & n.s. \\
\hline Sharp $(3 / 3)$ & $r=0.96 *$ & $r=0.97 *$ & $r=0.87 *$ \\
\hline
\end{tabular}

Acceleratior operation

Acceleratior operation
\begin{tabular}{|l|c|c|c|}
\hline & Real & Laboratory (Steering \& Pedals) & Laboratory (Game Pad) \\
\hline Sharp $(1 / 3)$ & $r=0.99 *$ & $r=0.87 *$ & $r=0.63 *$ \\
\hline Gentle $(1 / 2)$ & $r=0.98 *$ & $r=0.67 *$ & $r=0.84 *$ \\
\hline Straight $(1 / 2)$ & $r=0.95 *$ & $r=0.62 *$ & $r=0.86 *$ \\
\hline Sharp $(2 / 3)$ & $r=0.99 *$ & $r=0.78 *$ & $r=0.85 *$ \\
\hline Straight $(2 / 2)$ & $r=0.98 *$ & $r=0.88 *$ & $r=0.86 *$ \\
\hline Gentle $(2 / 2)$ & $r=0.99 *$ & n.s. & $r=0.91 *$ \\
\hline Sharp $(3 / 3)$ & $r=0.99 *$ & n.s. & $r=0.73 *$ \\
\hline
\end{tabular}

Acceleratior operation

\begin{tabular}{|l|c|c|c|}
\hline & Real & Laboratory (Steering \& Pedals) & Laboratory (Game Pad) \\
\hline Sharp (1/3) & $r=0.99 *$ & $r=0.54 *$ & $r=0.82 *$ \\
\hline Gentle $(1 / 2)$ & $r=0.94 *$ & $r=0.58 *$ & $r=0.75 *$ \\
\hline Straight $(1 / 2)$ & $r=0.94 *$ & $r=0.73 *$ & $r=0.94 *$ \\
\hline Sharp (2/3) & $r=0.98 *$ & $r=0.56 *$ & $r=0.83 *$ \\
\hline Straight $(2 / 2)$ & $r=0.94 *$ & $r=0.85 *$ & $r=0.85 *$ \\
\hline Gentle $(2 / 2)$ & $r=0.95 *$ & $r=0.82 *$ & $r=0.79 *$ \\
\hline Sharp $(3 / 3)$ & $r=0.99 *$ & $r=0.63 *$ & $r=0.56 *$ \\
\hline
\end{tabular}

Steering operation

Steering operation
\begin{tabular}{|l|c|c|c|}
\hline & Real & Laboratory (Steering \& Pedals) & Laboratory (Game Pad) \\
\hline Sharp (1/3) & $r=0.76 *$ & $r=0.90 *$ & $r=0.97 *$ \\
\hline Gentle $(1 / 2)$ & $r=0.79 *$ & $r=0.89 *$ & $r=0.90 *$ \\
\hline Straight $(1 / 2)$ & $r=0.99 *$ & $r=0.86 *$ & $r=0.89 *$ \\
\hline Sharp $(2 / 3)$ & $r=0.87 *$ & $r=0.95 *$ & $r=0.92 *$ \\
\hline Straight $(2 / 2)$ & $r=0.93 *$ & $r=0.71 *$ & $n . s$ \\
\hline Gentle $(2 / 2)$ & $r=0.93 *$ & $r=0.95 *$ & $r=0.94 *$ \\
\hline Sharp $(3 / 3)$ & $r=0.81 *$ & $r=0.94 *$ & $r=0.94 *$ \\
\hline
\end{tabular}
Steering operation

Steering operation
\begin{tabular}{|l|c|c|c|}
\hline & Real & Laboratory (Steering \& Pedals) & Laboratory (Game Pad) \\
\hline Sharp (1/3) & $r=0.82 *$ & $r=0.89 *$ & $r=0.92 *$ \\
\hline Gentle (1/2) & $r=0.77 *$ & $r=0.87 *$ & $r=0.91 *$ \\
\hline Straight (1/2) & $r=0.99 *$ & $r=0.50 *$ & $r=0.69 *$ \\
\hline Sharp (2/3) & $r=0.91 *$ & $r=0.87 *$ & $r=0.90 *$ \\
\hline Straight (2/2) & $r=0.85 *$ & $r=0.86 *$ & $r=0.72 *$ \\
\hline Gentle (2/2) & $r=0.93 *$ & $r=0.80 *$ & $r=0.89 *$ \\
\hline Sharp (3/3) & $r=0.85 *$ & $r=0.93 *$ & $r=0.82 *$ \\
\hline
\end{tabular}

Note: Significant correlations are marked by $*(p<.05)$. Table shows result of the Piason's correlations correlation analysis of between individual behaviors at in former three trials and the latter three trials. Columns indicate each system, and rows indicate each environment that corresponds to the course arrangement shown in Table 1. Tables on the left side show free driving situation results, and tables on right side show hurried rapid driving situation results.

The result suggests that even when there are many differences between systems from the viewpoint of ecological validity, behavioral consistency might remain in a specific situation where both a strong high-order cognitive constraint (i.e., rapid driving) and a weak low-order cognitive constraint (driving with easy handling toward a straight line course) were satisfied.

\section{Conclusion}

In this study, we discussed cross-situational consistency using an innovative experimental platform in which environment, system, and context factors were independently manipulated.

The results are summarized as follows. 1) The individual behaviors in each system were stable, and their consistency was retained. 2) The consistency of behaviors was also confirmed when the participants drove using different controllers as manipulation interfaces in the identical system. 3) However, only slight correlation was observed across different systems. We only observed significant consistency in a situation where a strong high-order cognitive constraint (i.e., rapid driving) and a weak low-order cognitive constraint (driving with easy handling toward a straight line course) were given.

We will conduct multivariate analysis to classify the individuality of the participant behaviors, and discuss cross-situational consistency from the viewpoint of individuality. Moreover, we are currently engaged in an experiment that includes a virtual system. These results will provide comprehensive analysis of cross-situational consistency. 
Table 3

Cross-situational consistency between systems: correlation between individual behaviors in different systems

Context. Free Driving
Brake operation
\begin{tabular}{|l|c|c|c|}
\hline & $\begin{array}{r}\text { Real } \times \text { Laboratory } \\
(\text { Steering \& Pedal) }\end{array}$ & $\begin{array}{r}\text { Real } \times \text { Laboratory } \\
(\text { Game Pad) }\end{array}$ & $\begin{array}{l}\text { Laboratory (Steering \& Pedals) } \\
\times \text { Laboratory (Game Pad) }\end{array}$ \\
\hline Sharp (1/3) & n.s. & n.s. & $r=0.79 *$ \\
\hline Gentle $(1 / 2)$ & n.s. & n.s. & $r=0.46 *$ \\
\hline Straight $(1 / 2)$ & n.s. & n.s. & n.s. \\
\hline Sharp $(2 / 3)$ & n.s. & n.s. & $r=0.42+$ \\
\hline Straight $(2 / 2)$ & n.s. & n.s. & n.s. \\
\hline Gentle (2/2) & n.s. & n.s. & n.s. \\
\hline Sharp $(3 / 3)$ & n.s. & n.s. & n.s. \\
\hline
\end{tabular}

Context. Rapid Driving
Brake operation
\begin{tabular}{|l|c|c|c|}
\hline & $\begin{array}{c}\text { Real } \times \text { Laboratory } \\
\text { (Steering \& Pedal) }\end{array}$ & $\begin{array}{r}\text { Real } \times \text { Laboratory } \\
\text { (Game Pad) }\end{array}$ & $\begin{array}{c}\text { Laboratory (Steering \& Pedals) } \\
\times \text { Laboratory (Game Pad) }\end{array}$ \\
\hline Sharp $(1 / 3)$ & n.s. & n.s. & n.s. \\
\hline Gentle $(1 / 2)$ & n.s. & n.s. & n.s. \\
\hline Straight $(1 / 2)$ & n.s. & $r=-0.39+$ & n.s. \\
\hline Sharp $(2 / 3)$ & n.s. & n.s. & $r=0.41+$ \\
\hline Straight $(2 / 2)$ & n.s. & n.s. & n.s. \\
\hline Gentle $(2 / 2)$ & n.s. & n.s. & n.s. \\
\hline Sharp $(3 / 3)$ & n.s. & n.s. & n.s. \\
\hline
\end{tabular}

Accelerator operation
\begin{tabular}{|l|c|c|c|}
\hline & $\begin{array}{r}\text { Real } \times \text { Laboratory } \\
\text { (Steering \& Pedal) }\end{array}$ & $\begin{array}{r}\text { Real } \times \text { Laboratory } \\
\text { (Game Pad) }\end{array}$ & $\begin{array}{c}\text { Laboratory (Steering \& Pedals) } \\
\times \text { Laboratory (Game Pad) }\end{array}$ \\
\hline Sharp (1/3) & n.s. & n.s. & n.s. \\
\hline Gentle (1/2) & n.s. & n.s. & $r=0.79 *$ \\
\hline Straight (1/2) & n.s. & n.s. & $r=0.72 *$ \\
\hline Sharp (2/3) & n.s. & n.s. & n.s. \\
\hline Straight (2/2) & n.s. & n.s. & $r=0.62 *$ \\
\hline Gentle (2/2) & n.s. & n.s. & n.s. \\
\hline Sharp (3/3) & n.s. & n.s. & n.s. \\
\hline
\end{tabular}

Accelerator operation
\begin{tabular}{|l|c|c|c|}
\hline & $\begin{array}{c}\text { Real } \times \text { Laboratory } \\
\text { (Steering \& Pedal) }\end{array}$ & $\begin{array}{r}\text { Real } \times \text { Laboratory } \\
\text { (Game Pad) }\end{array}$ & $\begin{array}{c}\text { Laboratory (Steering \& Pedals) } \\
\times \text { Laboratory (Game Pad) }\end{array}$ \\
\hline Sharp (1/3) & n.s. & n.s. & $r=0.42+$ \\
\hline Gentle $(1 / 2)$ & $r=0.53 *$ & $r=0.45 *$ & $r=0.62 *$ \\
\hline Straight $(1 / 2)$ & $r=0.43+$ & n.s. & $r=0.70 *$ \\
\hline Sharp (2/3) & n.s. & n.s. & n.s. \\
\hline Straight (2/2) & n.s. & n.s. & $r=0.43 *$ \\
\hline Gentle (2/2) & $r=0.46 *$ & n.s. & n.s. \\
\hline Sharp (3/3) & n.s. & n.s. & n.s. \\
\hline
\end{tabular}

Steering operation
\begin{tabular}{|l|c|c|c|}
\hline & $\begin{array}{r}\text { Real } \times \text { Laboratory } \\
\text { (Steering \& Pedal) }\end{array}$ & $\begin{array}{r}\text { Real } \times \text { Laboratory } \\
\text { (Game Pad) }\end{array}$ & $\begin{array}{c}\text { Laboratory (Steering \& Pedals) } \\
\times \text { Laboratory (Game Pad) }\end{array}$ \\
\hline Sharp (1/3) & n.s. & n.s. & $r=0.58 *$ \\
\hline Gentle (1/2) & n.s. & n.s. & $r=0.55 *$ \\
\hline Straight (1/2) & n.s. & $r=0.46 *$ & $r=0.59 *$ \\
\hline Sharp (2/3) & n.s. & n.s. & $r=0.56 *$ \\
\hline Straight (2/2) & n.s. & $r=0.54 *$ & $r=0.61 *$ \\
\hline Gentle (2/2) & n.s. & n.s. & $r=0.72 *$ \\
\hline Sharp (3/3) & n.s. & n.s. & $r=0.49 *$ \\
\hline
\end{tabular}

\begin{tabular}{|c|c|c|c|}
\hline \multicolumn{4}{|c|}{ Steering operation } \\
\hline & \begin{tabular}{|c|} 
Real $\times$ Laboratory \\
(Steering \& Pedal)
\end{tabular} & \begin{tabular}{|r|} 
Real x Laboratory \\
(Game Pad)
\end{tabular} & $\begin{array}{c}\text { Laboratory (Steering \& Pedals) } \\
\text { x Laboratory (Game Pad) }\end{array}$ \\
\hline Sharp (1/3) & n.s. & n.s. & $r=0.71 *$ \\
\hline Gentle (1/2) & n.s. & n.s. & $r=0.65 *$ \\
\hline Straight $(1 / 2)$ & n.s. & n.s. & $r=0.62 *$ \\
\hline Sharp $(2 / 3)$ & n.s. & n.s. & $r=0.63 *$ \\
\hline Straight $(2 / 2)$ & $r=0.39+$ & n.s. & n.s. \\
\hline Gentle (2/2) & n.s. & n.s. & $r=0.63 *$ \\
\hline Sharp $(3 / 3)$ & n.s. & $r=-0.46 *$ & $r=0.64 *$ \\
\hline
\end{tabular}

Note: Significant correlations are marked by $*(p<.05)$ or $+(p<.10)$. Table shows result of the Piason's correlation analysis of between individual behaviors between systems. Columns indicate combination of controllers of laboratory system and different systems, and rows indicate each environment. Tables on left side show free driving situation results, and tables in right side show hurried rapid driving situation result.

\section{References}

[1] C. A. Anderson, J. J. Lindsay, and B. J. Bushman, Research in the psychological laboratory: truth or triviality? Current Directions in Psychological Science, 1999, 8, 3-9.

[2] D. C. Funder, Towards a resolution of the personality triad: Persons, situations, and behaviors, Journal of Research in Personality, 2006, 40, 21-34.

[3] E. Brunswik, Perception and the representative design of psychological experiments, University of California Press, 1956.

[4] J. C. F. de Winter, S. de Groot, M. Mulder, P. A. Wieringa, J. Dankelman, and J. A. Mulder, Relationships between driving simulator performance and driving test results, Ergonomics, 2009, 52, 137-153.
[5] J. D. Lee, D. V. McGehee, T. L. Brown, and M. L. Reyes, Collision warning timing, driver distraction, and driver response to imminent rear-end collisions in a High-Fidelity driving simulator, Human Factors, 2002, 44, 314-334.

[6] O. Shechtman, S. Classen, K. Awadzi, and W. Mann, Comparison of driving error between on-the-road and simulated driving assessment: a validation study, Traffic Injury Prevention, 2009, 10, 379-385.

[7] R. M. Furr, Personality psychology as a truly behavioural science, European Journal of Personality, 2009, 23, 369-401.

[8] T. D. Cook and D. T. Campbell, Quasi-Experimentation: Design and Analysis for Field Settings, Rand McNally, Chicago, Illinois, 1979.

[9] W. Mischel and P. K. Peake, Beyond déjà vu in the search for cross-situational consistency. Psychological Review, 1982, 89, 730-755. 